\title{
Spontaneous hepatic haemangioma rupture and hemoperitoneum: a double problem with a single stage interventional radiology solution
}

\author{
Umberto G. Rossi ${ }^{1}$, Nicola Camisassi ${ }^{1}$, Francesco Pinna ${ }^{1}$, \\ Gian Andrea Rollandi
}

Department of Imaging Diagnosis, 'Interventional Radiology and ${ }^{2}$ Diagnostic Radiology Unit, E.O. Galliera Hospital, Genova, Italy

Hepatic haemangioma is a congenital vascular malformation, considered the most common benign mesenchymal hepatic tumour. Spontaneous or traumatic rupture is the most severe complication. In case of rupture, surgical resection and enucleation, as a single therapy or after trans-arterial embolization are considered the treatments of choice. We report a case of spontaneous rupture of a hepatic haemangioma with massive hemoperitoneum successfully treated by percutaneous hepatic trans-arterial embolization and pelvic drainage alone.

Keywords Liver; Hemangioma; Embolization; Hemoperitoneum; Drainage

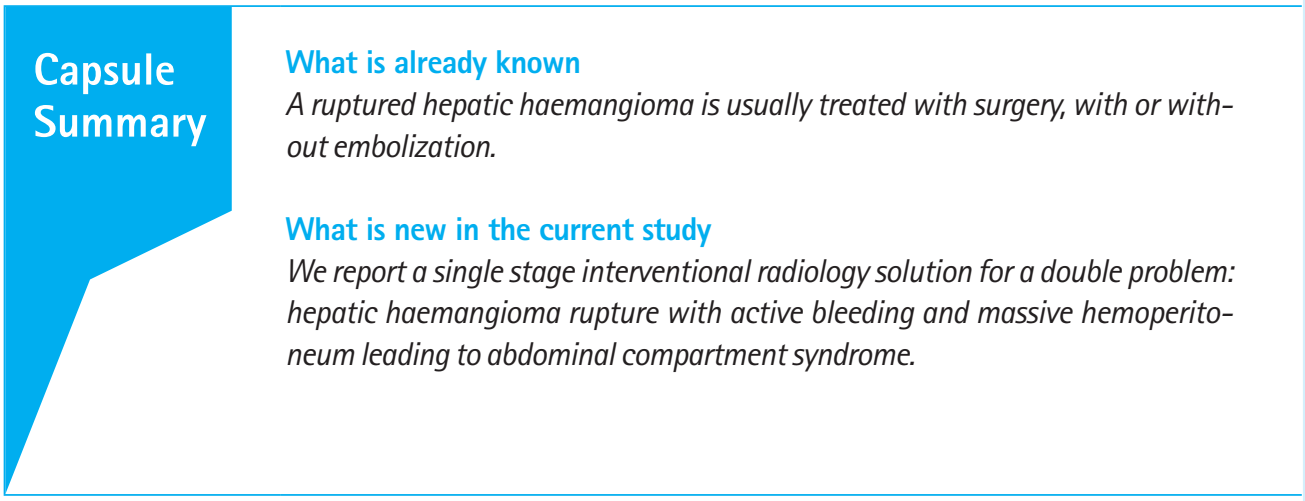

elSSN: 2383-4625

Received: 1 February 2018

Revised: 5 April 2018

Accepted: 18 April 2018

Correspondence to: Umberto G. Rossi Department of Imaging Diagnosis, Interventional Radiology Unit, E.O. Galliera Hospital, Mura delle Cappuccine, 14, Genova 16128, Italy E-mail: umberto.rossi@galliera.it ORCID

http://orcid.org/0000-0003-1869-8585

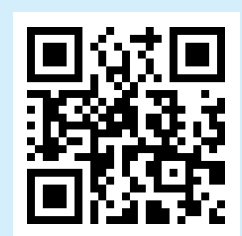

How to cite this article:

Rossi UG, Camisassi N, Pinna F, Rollandi GA. Spontaneous hepatic haemangioma rupture and hemoperitoneum: a double problem with a single stage interventional radiology solution. Clin Exp Emerg Med 2019;6(2):169172.

This is an Open Access article distributed under the terms of the Creative Commons Attribution Non-Commercial License (http:// creativecommons.org/licenses/by-nc/4.0/). 


\section{INTRODUCTION}

Hepatic haemangioma $(\mathrm{HH})$ is considered the most common benign mesenchymal tumour of the liver. ${ }^{1}$ It is composed of masses of blood vessels that are atypical or irregular in arrangement and size. Most cases of HHs are asymptomatic although a few patients may present with a wide variety of clinical symptoms. Spontaneous or traumatic HH rupture is considered the most severe complication. ${ }^{1,2}$ Here, we report a case of spontaneous rupture of a hepatic giant $\mathrm{HH}$ with massive hemoperitoneum that was treated by transcatheter arterial embolization (TAE) and placement of a percutaneous pelvic drainage.

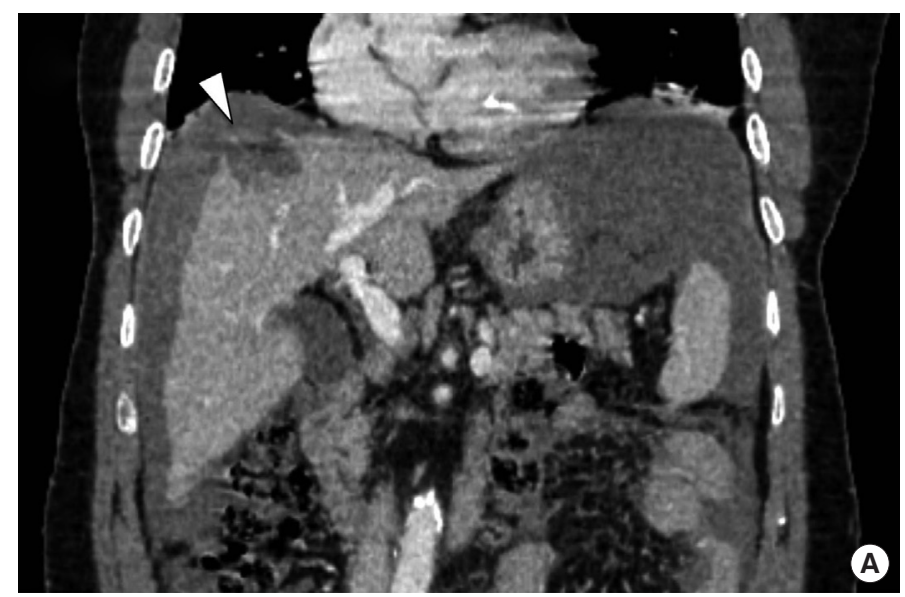

\section{CASE REPORT}

A 64-year-old male patient presented to our emergency department for upper right abdominal pain with tenderness. He denied any traumatic event and significant diseases in the past. Physical examination revealed a heart rate of 72 beats/min with a blood pressure of $98 / 46 \mathrm{mmHg}$. Laboratory investigation revealed a haemoglobin of $8.4 \mathrm{~g} / \mathrm{dL}$ and normal coagulopathy parameters and alpha-fetoprotein. He underwent urgent multi-detector computed tomography (MD-CT) that demonstrated a $5.1-\mathrm{cm} \mathrm{HH}$ located in segments VII and VIII, with active bleeding and associated massive hemoperitoneum (Fig. 1). After multidisciplinary discussion

Fig. 1. (A) Multi-detector computed tomography coronal multiplanar reconstruction that demonstrates liver rupture (arrowhead) due to a hepatic haemangioma located in segments VII and VIII. (B) Multi-detector computed tomography axial image at the level of the iliac fossa that shows massive hemoperitoneum (asterisk).
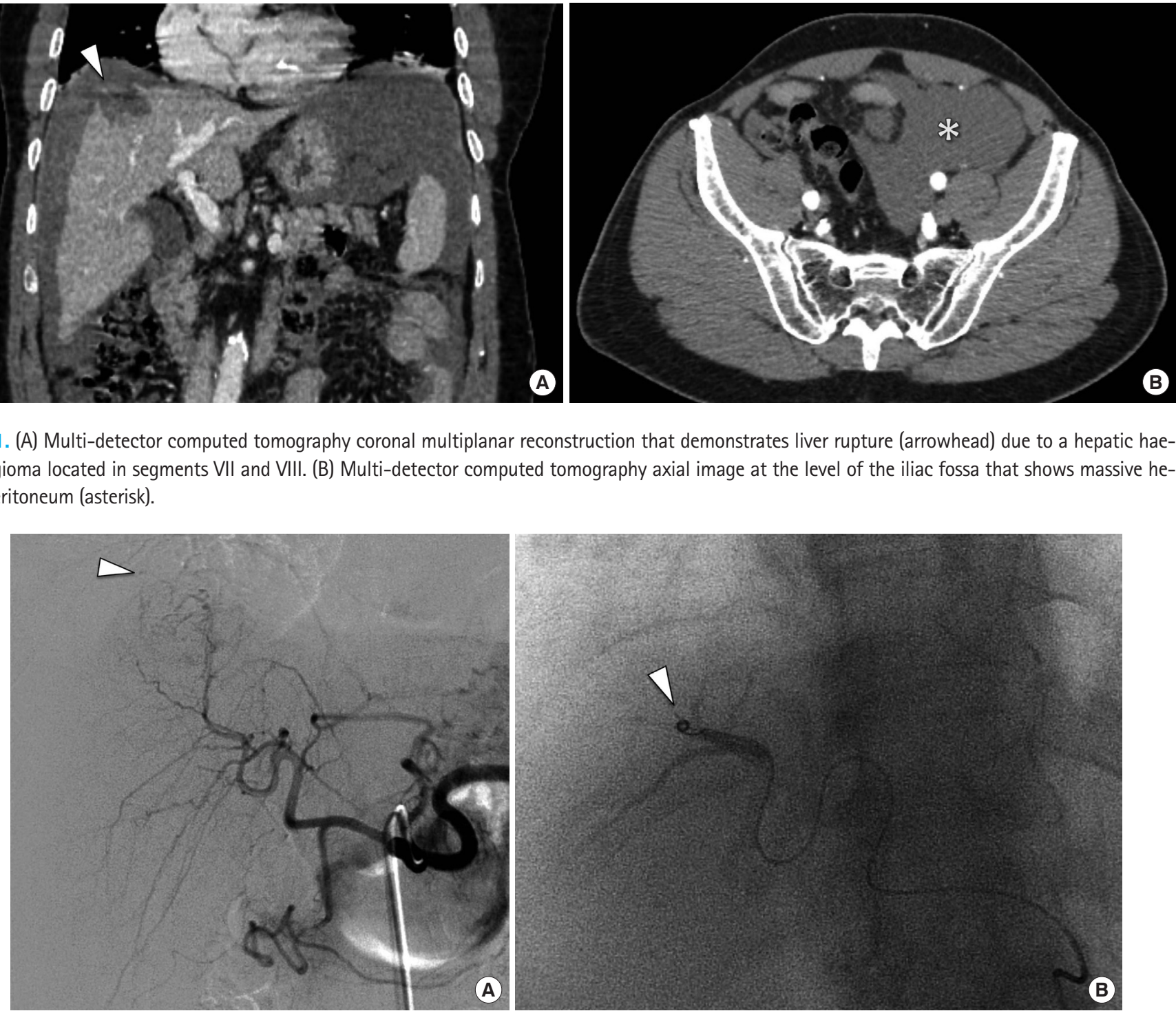

Fig. 2. (A) Digital subtraction angiography that confirms the active bleeding (arrowhead) from the hepatic haemangioma located in segments VII and VIII. (B) Angiographic control after super-selective embolization with micro-particles and metallic coils (arrowhead) of the right segmental hepatic arteries proximal to the hepatic haemangioma. 


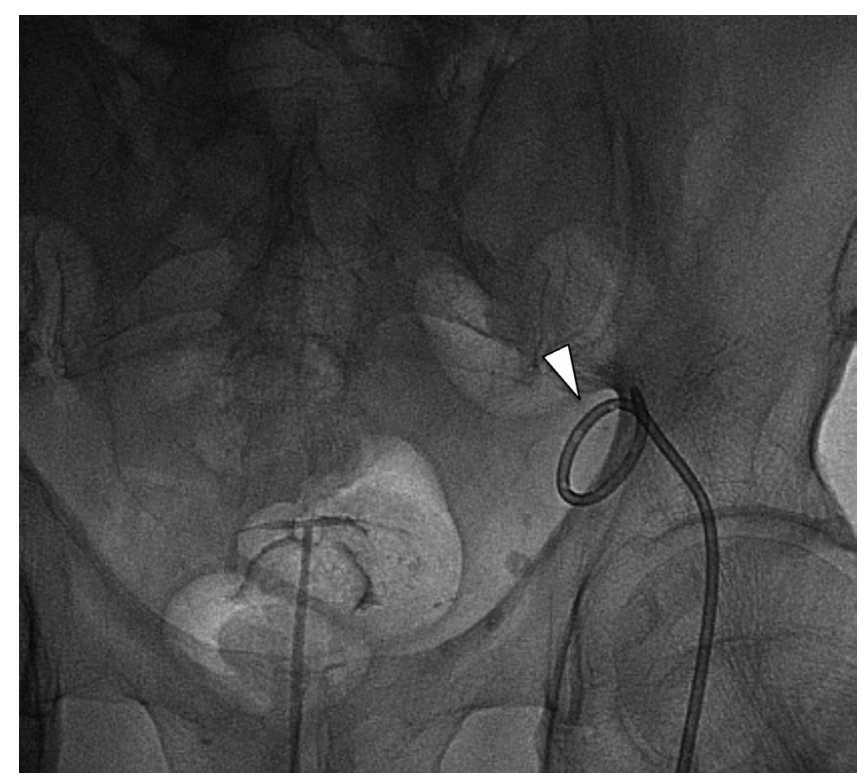

Fig. 3. Conventional radiology image that shows the 12-Fr catheter inserted through the left iliac fossa (arrowhead).

and agreement, a percutaneous approach with hepatic TAE and pelvic drainage was considered the first line treatment. Surgery was considered only if the percutaneous approach failed or had recurrence. The patient underwent urgent digital subtraction angiography of the common hepatic artery that confirmed the site of active bleeding from the $\mathrm{HH}$ into the VII and VIII segments (Fig. 2A). After coaxial super-selective catheterization with a coaxial 2.7-Fr micro-catheter (Progreat, Terumo Corp., Tokyo, Japan) of the two main feeding arteries of the $\mathrm{HH}$ from the right hepatic arteries, the active bleeding was embolized with 400 micron micro-particles (Embozene, Boston Scientific, Natick, MA, USA) and two 3-mm metallic micro-coils (VortX, Boston Scientific) (Fig. 2B). The intra-abdominal pressure was increased to a value of 21 $\mathrm{mmHg}$ (grade III). So, under ultrasound guidance a 12-Fr catheter (Boston Scientific) was inserted using Seldinger's technique into the left iliac fossa (Fig. 3). About 1.6 L of altered blood was drained over the next hour. There was no recurrence or complication; therefore surgery was not performed since embolization proved to be effective and definitive treatment. On day ten of hospitalization, the patient underwent MD-CT that revealed absence of active bleeding, correct hepatic embolization and absence of intra-abdominal collections. Then he was discharged. Follow-up by clinical examination and MD-CT (Fig. 4) at six months demonstrated the success of the percutaneous approach and no signs of bleeding recurrence.

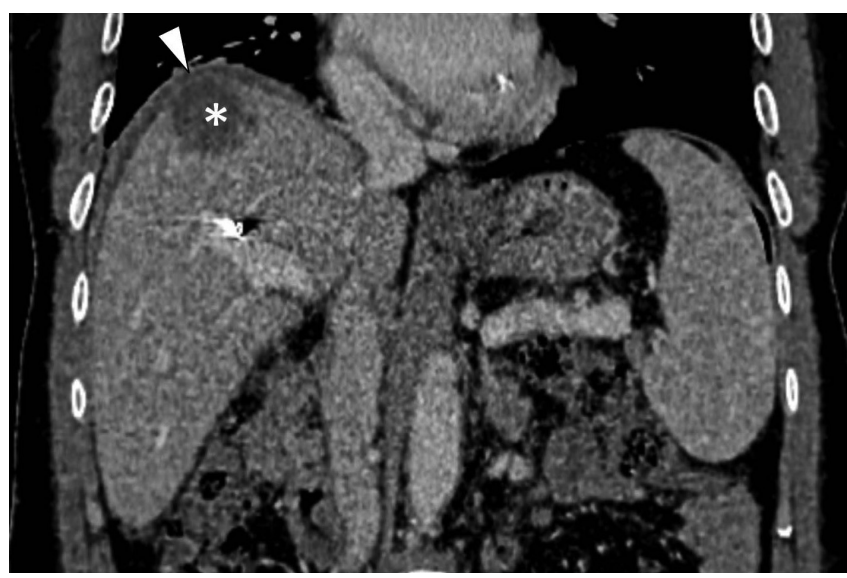

Fig. 4. Multi-detector computed tomography coronal multiplanar reconstruction at six months that demonstrates the repaired integrity of the liver capsule (arrowhead) due to a hepatic haemangioma located in segments VII and VIII as prevalent hypo-dense formation (asterisk).

\section{DISCUSSION}

HHs are classified as primary benign vascular tumours of the liver, and can be divided into two major groups: capillary haemangioma (generally small and sometimes multiple), and cavernous haemangioma (large and defined as giant when larger than $5 \mathrm{~cm}$ ). ${ }^{1}$ $\mathrm{HH}$ prevalence is between $0.4 \%$ to $7.3 \%$ and occurs most frequently adults in their fourth-fifth decades of life. ${ }^{1,2}$ As in most cases $\mathrm{HHs}$ are asymptomatic, they are usually diagnosed as incidental findings on imaging studies of the abdomen. ${ }^{2}$ When they grow they can cause right upper abdominal pain due to hepatic capsular distension or compression of adjacent structures. ${ }^{1-3}$ Risks for $\mathrm{HH}$ rupture include: size $>5 \mathrm{~cm}$, growth over time, and subglissonian localization. ${ }^{2}$

Spontaneous HH hemorrhage is an uncommon condition. But when it occurs, clinical manifestations consist of sudden abdominal pain and hypovolemic shock in about one third of cases. ${ }^{1-3}$ Its treatment is considered a life-saving manoeuvre. The treatments for spontaneous HH with active hemorrhage are surgical lobectomy, surgical packing or TAE. ${ }^{14-6}$ TAE has become a more common treatment in selected cases of spontaneous, blunt and/or penetrating liver rupture improving overall patient outcomes. ${ }^{4-6}$ The advantage of the TAE is the mini-invasiveness of the procedure compared to open surgery. However, the use of TAE alone, as an alternative to surgery, in the management of ruptured liver nodules is controversial due to its possible complications: persistent intracavitary bleeding and partial liver ischemia with possible susequent infection. ${ }^{14-6}$ This is why every case where TAE is used as a single and definitive therapy should be discussed and evaluated by a multidisciplinary team. It should be remembered that if TAE is not ther- 
apeutic, it does not preclude a second open surgical treatment. In our case the super-selective embolization and the minimal rupture of the hepatic capsule led to the complete success of TAE alone. Several embolic agents have been used such as: particles, coils, liquid embolic agents, and glue. ${ }^{1,3-7}$ In our case we have used two different embolic materials: particles for distal vessel occlusion and coils for the segmental part of the intra-hepatic artery. The real limitation of TAE is that it does not allow a histological diagnosis of the lesion after the treatment. Therefore an imaging and laboratory preliminary and follow-up evaluation is required to confirm the absence of frank malignancy.

When acute hepatic active bleeding is solved by TAE, associated hemoperitoneum has to be evaluated. When it is massive, it leads to abdominal compartment syndrome (ACS) which is a disorder caused by rapidly increasing intra-abdominal pressure. ACS is divided into four grades: grade I 12 to $15 \mathrm{mmHg}$, grade II 16 to $20 \mathrm{mmHg}$, grade III 21 to $25 \mathrm{mmHg}$, and grade IV > $25 \mathrm{mmHg}^{8}$ Generally its treatment of choice is surgical decompression., ${ }^{9,10}$ In our case performance of percutaneous pelvic drainage, before blood had started clotting, guaranteed the possibility of its removal and therefore the resolution of the ACS.

Nowadays, in selected cases of $\mathrm{HH}$ rupture with hemoperitoneum, TAE and imaging guided percutaneous pelvic drainage can be performed as an alternative to surgery. This technique can guarantee an equally effective therapy with reduced invasiveness.

\section{CONFLICT OF INTEREST}

No potential conflict of interest relevant to this article was reported.

\section{REFERENCES}

1. Ribeiro MA Jr, Papaiordanou F, Goncalves JM, Chaib E. Spon- taneous rupture of hepatic hemangiomas: a review of the literature. World J Hepatol 2010;2:428-33.

2. Mocchegiani $F$, Vincenzi $P$, Coletta $M$, et al. Prevalence and clinical outcome of hepatic haemangioma with specific reference to the risk of rupture: a large retrospective cross-sectional study. Dig Liver Dis 2016;48:309-14.

3. Aiura K, Ohshima R, Matsumoto K, et al. Spontaneous rupture of liver hemangioma: risk factors for rupture. J Hepatobiliary Pancreat Surg 1996;3:308-12.

4. Yang JW, Li Y, Xie K, et al. Spontaneous rupture of hepatic epithelioid hemangioendothelioma: a case report. World J Gastroenterol 2017;23:185-90.

5. Yamamoto $T$, Kawarada $Y$, Yano $T$, Noguchi $T$, Mizumoto $R$. Spontaneous rupture of hemangioma of the liver: treatment with transcatheter hepatic arterial embolization. Am J Gastroenterol 1991;86:1645-9.

6. Vassiou K, Rountas H, Liakou P, et al. Embolization of a giant hepatic hemangioma prior to urgent liver resection. Case report and review of the literature. Cardiovasc Intervent Radiol 2007;30:800-2.

7. Rossi UG, Cariati M. Transarterial embolization for hepatocellular carcinoma: reasoning on mechanisms of action, tips and tricks of the procedure. Eur J Gastroenterol Hepatol 2017;29: 488-9.

8. Malbrain $M L$, Cheatham $M L$, Kirkpatrick $A$, et al. Results from the International Conference of Experts on Intra-abdominal Hypertension and Abdominal Compartment Syndrome. I. Definitions. Intensive Care Med 2006;32:1722-32.

9. Vikrama KS, Shyamkumar NK, Vinu M, et al. Percutaneous catheter drainage in the treatment of abdominal compartment syndrome. Can J Surg 2009;52:E19-20.

10. Standl T. Abdominal compartment syndrome: a still underestimated problem? Anasthesiol Intensivmed Notfallmed Schmerzther 2007;42:500-3. 